\title{
Dynamic Behavior of a One-Dimensional Wave Equation with Memory and Viscous Damping
}

\author{
Jing Wang \\ School of Mathematics, Beijing Institute of Technology, Beijing 100081, China \\ Correspondence should be addressed to Jing Wang; wangjlele@163.com
}

Received 9 January 2014; Accepted 17 March 2014; Published 1 April 2014

Academic Editors: Y. Dimakopoulos, M. Hermann, and M.-H. Hsu

Copyright (C) 2014 Jing Wang. This is an open access article distributed under the Creative Commons Attribution License, which permits unrestricted use, distribution, and reproduction in any medium, provided the original work is properly cited.

\begin{abstract}
We study the dynamic behavior of a one-dimensional wave equation with both exponential polynomial kernel memory and viscous damping under the Dirichlet boundary condition. By introducing some new variables, the time-variant system is changed into a time-invariant one. The detailed spectral analysis is presented. It is shown that all eigenvalues of the system approach a line that is parallel to the imaginary axis. The residual and continuous spectral sets are shown to be empty. The main result is the spectrum-determined growth condition that is one of the most difficult problems for infinite-dimensional systems. Consequently, an exponential stability is concluded.
\end{abstract}

\section{Introduction}

It is known that viscoelastic materials have been widely used in mechanics, chemical engineering, architecture, traffic, information, and so on. More and more researchers have paid close attention to the dynamic behavior and control of vibration for elastic structures with viscoelasticity over the past several decades. The most widely used models for viscoelasticity are the Boltzmann model and Kelvin-Voigt model. For instance, the results concerning the exponential asymptotic stability of a linear hyperbolic integrodifferential equation in Hilbert space are established in [1], which is an abstract version of the equation of motion for dynamic linear viscoelastic solids. In $[2,3]$, the exponential stabilities of a vibrating beam with one segment made of viscoelastic material of a Kelvin-Voigt type and a vibrating string with Boltzmann damping are proved under certain hypotheses of the smoothness and structural condition of the coefficients of the system. In [4], the global existence and exponential decay of solutions of a nonlinear unidimensional wave equation with a viscoelastic boundary condition are analyzed under some assumptions. Spectral analyses of a wave equation with internal Kelvin-Voigt damping and Boltzmann damping are considered in $[5,6]$, respectively. The Riesz basis property of the generalized eigenfunctions of a one-dimensional hyperbolic system which is a heat equation incorporating the effect of thermomechanical coupling and the effect of inertia is studied in [7]. In [8], a detailed spectral analysis for a heat equation system which is derived from a thermoelastic equation with memory type is presented and the spectrumdetermined growth condition and strong exponential stability are then concluded. Similar studies from different aspects for elastic structures with viscoelasticity can also be found in [9-14] and the references therein.

In this paper, we are interested in the following onedimensional wave equation with viscoelastic damping under the Dirichlet boundary condition:

$$
\begin{gathered}
w_{t t}(x, t)=a^{2} w_{x x}(x, t)+\int_{0}^{t} \kappa(t-s) w_{x}(x, s) d s \\
-c w_{t}(x, t), \quad 0<x<1, t>0, \\
w(0, t)=w(1, t)=0, \quad t>0, \\
w(x, 0)=w_{0}(x), \quad w_{t}(x, 0)=w_{1}(x), \quad 0<x<1,
\end{gathered}
$$


where $a$ and $c$ are positive constants and the kernel is taken as the finite sum of exponential polynomials:

$$
\kappa(t)=\sum_{i=1}^{N} a_{i} e^{-b_{i} t}, \quad 0<a_{i}, \quad b_{i} \in \mathscr{R}, 1 \leq N \in \mathcal{N},
$$

and we assume that, for simplicity,

$$
0<b_{1}<b_{2}<\cdots<b_{N}
$$

Our interest is to investigate the dynamic behavior of (1)-(2), particularly the large time behavior. The asymptotic distribution of eigenvalues of the system is discussed in detail. It is shown that the system operator is of compact resolvent and hence its spectrum consists of isolated eigenvalues of finite algebraic multiplicity only. All eigenvalues approach a line that is parallel to the imaginary axis. The main result is the spectrum-determined growth condition which is one of the most difficult problems for infinite-dimensional systems. Consequently, a strongly exponential stability is concluded.

The paper is organized as follows. In Section 2, we introduce some new variables so that the system (1) with kernel (2) is reduced to be a time-invariant one. Section 3 is devoted to the detailed spectral analysis of the newly formulated system. The main result is the spectrum-determined growth condition that is presented in Section 4; finally, a strongly exponential stability is obtained.

\section{System Operator Setup}

Introduce

$$
\varphi_{i}(x, t)=a_{i} \int_{0}^{t} e^{-b_{i}(t-s)} w(x, s) d s, \quad i=1,2, \ldots, N .
$$

Then $\varphi_{i}$ satisfies

$$
\begin{gathered}
\left(\varphi_{i}\right)_{t}(x, t)=a_{i} w(x, t)-b_{i} \varphi_{i}(x, t), \\
\left(\varphi_{i}\right)_{x}(x, t)=a_{i} \int_{0}^{t} e^{-b_{i}(t-s)} w_{x}(x, s) d s \\
\varphi_{i}(x, 0)=0 .
\end{gathered}
$$

Thus, we can rewrite the systems (1) and (2) as

$$
\begin{gathered}
w_{t t}(x, t)-a^{2} w_{x x}(x, t)-\frac{\partial}{\partial x}\left[\sum_{j=1}^{N} \varphi_{i}(x, t)\right] \\
+c w_{t}(x, t)=0, \quad x \in(0,1), t>0, \\
\left(\varphi_{i}\right)_{t}(x, t)=a_{i} w(x, t)-b_{i} \varphi_{i}(x, t), \quad i=1,2, \ldots, N, \\
w(0, t)=w(1, t)=0, \quad t>0, \\
w(x, 0)=w_{0}(x), \quad w_{t}(x, 0)=w_{1}(x), \quad \varphi_{i}(x, 0)=0, \\
x \in(0,1), \quad i=1,2, \ldots, N .
\end{gathered}
$$

Obviously, the system (6) is a time-invariant system. The energy function of (6) is given by

$$
\begin{aligned}
E(t)=\frac{1}{2} \int_{0}^{1}[ & a^{2}\left|w_{x}(x, t)\right|^{2}+\left|w_{t}(x, t)\right|^{2} \\
& \left.+\sum_{i=1}^{N}\left(\left|\varphi_{i}^{\prime}(x, t)\right|^{2}+\left|\varphi_{i}(x, t)\right|^{2}\right)\right] d x .
\end{aligned}
$$

Motivated by the energy function, it is natural to consider the system (6) in the following Hilbert space $\mathscr{H}$ :

$$
\mathscr{H}=H_{0}^{1}(0,1) \times L^{2}(0,1) \times\left(H^{1}(0,1)\right)^{N},
$$

equipped with the inner product, $\forall\left(w, v, \varphi_{1}, \ldots, \varphi_{N}\right)$, $\left(f, g, h_{1}, \ldots, h_{N}\right) \in \mathscr{H}$

$$
\begin{aligned}
\left\langle\left(w, v, \varphi_{1}, \ldots, \varphi_{N}\right),\left(f, g, h_{1}, \ldots, h_{N}\right)\right\rangle_{1} \\
=\int_{0}^{1} a^{2} w^{\prime}(x) \overline{f^{\prime}(x)} d x+\int_{0}^{1} v(x) \overline{g(x)} d x \\
\quad+\sum_{i=1}^{N} \int_{0}^{1}\left[\varphi_{i}^{\prime}(x) \overline{h_{i}^{\prime}}(x)+\varphi_{i}(x) \overline{h_{i}}(x)\right] d x .
\end{aligned}
$$

Now, define the system operator $\mathscr{A}: D(\mathscr{A})(\subset \mathscr{H}) \rightarrow \mathscr{H}$ by

$$
\mathscr{A}\left(\begin{array}{c}
w \\
v \\
\varphi_{1} \\
\vdots \\
\varphi_{N}
\end{array}\right)^{\top}=\left(\begin{array}{c}
v \\
a^{2} w^{\prime \prime}+\frac{d}{d x}\left(\sum_{i=1}^{N} \varphi_{i}\right)-c v \\
a_{1} w-b_{1} \varphi_{1} \\
\vdots \\
a_{N} w-b_{N} \varphi_{N}
\end{array}\right)^{\top}
$$

$D(\mathscr{A})$

$$
=\left\{\left[w, v, \varphi_{1}, \ldots, \varphi_{N}\right] \in \mathscr{H}_{1} \mid v \in H_{0}^{1}(0,1), w^{\prime \prime} \in L^{2}(0,1)\right\} .
$$

Then (6) can be formulated as an abstract evolution equation in $\mathscr{H}$ as

$$
\frac{d}{d t} Y(t)=\mathscr{A} Y(t), \quad Y(0)=Y_{0}
$$

where $Y(t)=\left(w(\cdot, t), w_{t}(\cdot, t), \varphi_{1}(\cdot, t), \ldots, \varphi_{N}(\cdot, t)\right)$ is the state variable and $Y_{0}=\left(w_{0}(\cdot), w_{1}(\cdot), 0, \ldots, 0\right)$ is the initial value.

Lemma 1. Let $\mathscr{A}$ be defined by (10). Then $\mathscr{A}^{-1}$ exists and hence $0 \in \rho(\mathscr{A})$, the resolvent set of $\mathscr{A}$. Moreover, $\mathscr{A}$ generates a $C_{0^{-}}$ semigroup on $\mathscr{H}$. 
Proof. Let $\tilde{Y}=\left(\widetilde{w}, \widetilde{v}, \widetilde{\varphi_{1}}, \ldots, \widetilde{\varphi_{N}}\right) \in \mathscr{H}$. Solve $\mathscr{A} Y=\widetilde{Y}$ for $Y=\left(w, v, \varphi_{1}, \ldots, \varphi_{N}\right) \in D(\mathscr{A})$; that is,

$$
\begin{gathered}
v(x)=\widetilde{w}(x), \\
a^{2} w^{\prime \prime}(x)+\frac{d}{d x}\left(\sum_{i=1}^{N} \varphi_{i}(x)\right)-c v(x)=\widetilde{v}(x), \\
a_{i} w(x)-b_{i} \varphi_{i}(x)=\widetilde{\varphi}_{i}(x), \quad i=1,2, \ldots, N, \\
w(0)=w(1)=0,
\end{gathered}
$$

to give

$$
\begin{gathered}
v(x)=\widetilde{w}(x), \\
\varphi_{i}(x)=\frac{a_{i}}{b_{i}} w(x)-\frac{1}{b_{i}} \widetilde{\varphi}_{i}(x), \quad i=1,2, \ldots, N, \\
w^{\prime \prime}(x)+\frac{1}{a^{2}} \sum_{i=1}^{N} \frac{a_{i}}{b_{i}} w^{\prime}(x)=\frac{1}{a^{2}}\left(c \widetilde{w}(x)+\widetilde{v}(x)+\sum_{i=1}^{N} \frac{\widetilde{\varphi}_{i}^{\prime}(x)}{b_{i}}\right) .
\end{gathered}
$$

Set $\widetilde{G}(x)=c \widetilde{w}(x)+\widetilde{v}(x)+\sum_{i=1}^{N} \widetilde{\varphi}_{i}^{\prime}(x) / b_{i}$, together with the boundary condition $w(0)=w(1)=0$ to obtain

$$
\begin{aligned}
w(x)=\frac{1}{A}[ & C\left(1-e^{-\left(A / a^{2}\right) x}\right) \\
& \left.+\int_{0}^{x}\left(1-e^{-\left(A / a^{2}\right)(x-\tau)}\right) \widetilde{G}(\tau) d \tau\right],
\end{aligned}
$$

where

$$
\begin{array}{r}
C=\left(e^{-A / a^{2}}-1\right)^{-1} \int_{0}^{1}\left(1-e^{-\left(A / a^{2}\right)(1-\tau)}\right) \widetilde{G}(\tau) d \tau, \\
A=\sum_{i=1}^{N} \frac{a_{i}}{b_{i}} .
\end{array}
$$

Collecting (13), (15), and (16), we get the unique solution $Y$ to (12). Hence, $Y \in D(\mathscr{A})$ and $\mathscr{A}^{-1}$ exists, or $0 \in \rho(\mathscr{A})$.

Now, we show that $\mathscr{A}$ can generate a $C_{0}$-semigroup on $\mathscr{H}$. Actually, for any $=\left(w, v, \varphi_{1}, \ldots, \varphi_{N}\right) \in D(\mathscr{A})$, we have $\langle\mathscr{A} Y, Y\rangle_{1}$

$$
\begin{aligned}
& =\left\langle\left(\begin{array}{c}
v \\
a^{2} w^{\prime \prime}+\frac{d}{d x}\left(\sum_{i=1}^{N} \varphi_{i}\right)-c v \\
a_{1} w-b_{1} \varphi_{1} \\
\vdots \\
a_{N} w-b_{N} \varphi_{N}
\end{array}\right)^{\top},\left(\begin{array}{c}
w \\
v \\
\varphi_{1} \\
\vdots \\
\varphi_{N}
\end{array}\right)^{\top}\right\rangle \\
= & a^{2}\left\langle v^{\prime}, w^{\prime}\right\rangle_{L^{2}}+\left\langle a^{2} w^{\prime \prime}+\left(\sum_{i=1}^{N} \varphi_{i}\right)^{\prime}-c v, v\right\rangle_{L^{2}} \\
& +\sum_{i=1}^{N}\left\langle a_{i} w^{\prime}-b_{i} \varphi_{i}^{\prime}, \varphi_{i}^{\prime}\right\rangle_{L^{2}}+\sum_{i=1}^{N}\left\langle a_{i} w-b_{i} \varphi_{i}, \varphi_{i}\right\rangle_{L^{2}}
\end{aligned}
$$

$$
\begin{aligned}
= & -a^{2}\left\langle v, w^{\prime \prime}\right\rangle_{L^{2}}+a^{2}\left\langle w^{\prime \prime}, v\right\rangle_{L^{2}} \\
& +\left\langle\left(\sum_{i=1}^{N} \varphi_{i}\right)^{\prime}, v\right\rangle_{L^{2}}-c\|v\|_{L^{2}}^{2} \\
& +\sum_{i=1}^{N} a_{i}\left\langle w^{\prime}, \varphi_{i}^{\prime}\right\rangle_{L^{2}}+\sum_{i=1}^{N} a_{i}\left\langle w, \varphi_{i}\right\rangle_{L^{2}} \\
& -\sum_{i=1}^{N} b_{i}\left\|\varphi_{i}^{\prime}\right\|_{L^{2}}^{2}-\sum_{i=1}^{N} b_{i}\left\|\varphi_{i}\right\|_{L^{2}}^{2},
\end{aligned}
$$

$\operatorname{Re}\langle A \mathcal{A}, Y\rangle$

$$
\begin{aligned}
= & \operatorname{Re}\left(\left\langle\left(\sum_{i=1}^{N} \varphi_{i}\right)^{\prime}, v\right\rangle+\sum_{i=1}^{N} a_{i}\left\langle w^{\prime}, \varphi_{i}^{\prime}\right\rangle_{L^{2}}+\sum_{i=1}^{N} a_{i}\left\langle w, \varphi_{i}\right\rangle_{L^{2}}\right) \\
& -c\|v\|_{L^{2}}^{2}-\sum_{i=1}^{N} b_{i}\left\|\varphi_{i}^{\prime}\right\|_{L^{2}}^{2}-\sum_{i=1}^{N} b_{i}\left\|\varphi_{i}\right\|_{L^{2}}^{2} \\
\leq & -c\|v\|_{L^{2}}^{2}-\sum_{i=1}^{N} b_{i}\left\|\varphi_{i}^{\prime}\right\|_{L^{2}}^{2} \\
& -\sum_{i=1}^{N} b_{i}\left\|\varphi_{i}\right\|_{L^{2}}^{2}+\frac{N}{2}\|v\|_{L^{2}}^{2}+\frac{1}{2} \sum_{i=1}^{N}\left\|\varphi_{i}^{\prime}\right\|_{L^{2}}^{2} \\
& +\frac{N}{2}\left\|a w^{\prime}\right\|_{L^{2}}^{2}+\frac{1}{2} \sum_{i=1}^{N} \frac{a_{i}^{2}}{a^{2}}\left\|\varphi_{i}^{\prime}\right\|_{L^{2}}^{2}+\frac{N}{2}\|a w\|_{L^{2}}^{2} \\
& +\frac{1}{2} \sum_{i=1}^{N} \frac{a_{i}^{2}}{a^{2}}\left\|\varphi_{i}\right\|_{L^{2}}^{2} \\
\leq & N\left\|a w^{\prime}\right\|_{L^{2}}^{2}+\left(\frac{N}{2}-c\right)\|v\|_{L^{2}}^{2} \\
& +\sum_{i=1}^{N}\left(\frac{1}{2}+\frac{1}{2} \frac{a_{i}^{2}}{a^{2}}-b_{i}\right)\left\|\varphi_{i}^{\prime}\right\|_{L^{2}}^{2} \\
& +\sum_{i=1}^{N}\left(\frac{1}{2} \frac{a_{i}^{2}}{a^{2}}-b_{i}\right)\left\|\varphi_{i}\right\|_{L^{2}}^{2},
\end{aligned}
$$

where we have used the fact $\|a w\|_{L^{2}}^{2} \leq\left\|a w^{\prime}\right\|_{L^{2}}^{2}$. Let

$$
M=\max \left\{N, \frac{N}{2}-c, \frac{1}{2}+\frac{1}{2} \frac{a_{1}^{2}}{a^{2}}-b_{1}, \ldots, \frac{1}{2}+\frac{1}{2} \frac{a_{N}^{2}}{a^{2}}-b_{N}\right\} .
$$

Then we have

$$
\operatorname{Re}\langle\mathscr{A} Y, Y\rangle \leq M\langle Y, Y\rangle
$$

And, hence, $\operatorname{Re}\langle(\mathscr{A}-M I) Y, Y\rangle \leq 0$. Therefore, by the Lumer-Phillips theorem [15, p.14, Theorem 4.3], $(\mathscr{A}-M I)$ generates a $C_{0}$-semigroup of contractions on $\mathscr{H}$; thus $\mathscr{A}$ is an infinitesimal generator of a $C_{0}$-semigroup $e^{\mathscr{A} t}$ by the perturbation theory of semigroup [15, p.76, Theorem 1.1]. 


\section{Spectral Analysis of System Operator}

In this section, we investigate the distribution of spectrum of $\mathscr{A}$ in the complex plane. Firstly, we consider the eigenvalue problem.

Suppose that $\mathscr{A} Y=\lambda Y$ for $\lambda \in \mathscr{C}$ and $0 \neq Y=$ $\left(w, v, \varphi_{1}, \ldots, \varphi_{N}\right) \in D(\mathscr{A})$; that is,

$$
\begin{gathered}
v(x)=\lambda w(x), \\
a^{2} w^{\prime \prime}(x)+\frac{d}{d x}\left(\sum_{i=1}^{N} \varphi_{i}(x)\right)-c v(x)=\lambda v(x), \\
a_{i} w(x)-b_{i} \varphi_{i}(x)=\lambda \varphi_{i}(x), \quad i=1,2, \ldots, N, \\
w(0)=w(1)=0 .
\end{gathered}
$$

Proposition 2. Let $\mathscr{A}$ be defined as in (10). Then $\lambda=-b_{i}, i=$ $1,2, \ldots, N$, are eigenvalues of $\mathscr{A}$, which are corresponding to eigenfunctions $e_{i+2}, i=1,2, \ldots, N$, respectively, where $e_{i}$ is a constant function whose element is the ith element of the canonical basis of $\mathscr{R}^{N+2}$. Moreover, each of these eigenvalues is algebraically simple.

Proof. We only give the proof for $\lambda=-b_{1}$ because other cases can be treated similarly. Let $\lambda=-b_{1}$ and the eigenvalue problem becomes

$$
\begin{gathered}
v(x)=-b_{1} w(x), \\
a^{2} w^{\prime \prime}(x)+\frac{d}{d x}\left(\sum_{i=1}^{N} \varphi_{i}(x)\right)-c v(x)=-b_{1} v(x), \\
a_{1} w(x)=0, \\
a_{i} w(x)-b_{i} \varphi_{i}(x)=-b_{1} \varphi_{i}(x), \quad i=2, \ldots, N .
\end{gathered}
$$

From the third equation of (22), it has $w \equiv 0$. This in turn, together with the fourth equation of (22), yields

$$
\left(b_{1}-b_{i}\right) \varphi_{i}(x)=0, \quad i=2, \ldots, N,
$$

and, by (3), we arrive at

$$
\varphi_{i}(x)=0, \quad i=2, \ldots, N .
$$

By the first and the second equations of (22), these yield

$$
v(x)=0, \quad \varphi_{1}^{\prime}(x)=0, \quad 0<x<1 .
$$

Therefore, $e_{3}$ is an eigenfunction of $\mathscr{A}$ corresponding to $-b_{1}$. Further computation of $\left(\mathscr{A}+b_{1} I\right) F_{1}=-e_{3}$, where $F_{1}=$ $\left(g, h, \psi_{1}, \ldots, \psi_{N}\right) \in D(\mathscr{A})$, yields

$$
\begin{gathered}
h(x)+b_{1} g(x)=0, \quad 0<x<1, \\
a^{2} g^{\prime \prime}(x)+\frac{d}{d x}\left(\sum_{i=1}^{N} \psi_{i}(x)\right)+\left(b_{1}-c\right) h(x)=0, \quad 0<x<1, \\
a_{1} g(x)=-1, \quad 0<x<1, \\
\left(b_{1}-b_{i}\right) \psi_{i}(x)+a_{i} g(x)=0, \quad i=2, \ldots, N, \quad 0<x<1, \\
g(0)=g(1)=0 .
\end{gathered}
$$

We claim that (26) has no solution since $g$ must satisfy

$$
g(x)=-\frac{1}{a_{1}}, \quad g(0)=g(1)=0
$$

which is impossible. Hence $\lambda=-b_{1}$ is algebraically simple. The proof is complete.

When $\lambda \neq-b_{i}, i=1,2, \ldots, N$, it follows from (21) that

$$
\begin{gathered}
v(x)=\lambda w(x), \\
\varphi_{i}(x)=\frac{a_{i}}{\lambda+b_{i}} w(x), \quad i=1,2, \ldots, N .
\end{gathered}
$$

And $w$ satisfies

$$
\begin{gathered}
a^{2} w^{\prime \prime}(x)+\left(\sum_{i=1}^{N} \frac{a_{i}}{\lambda+b_{i}}\right) w^{\prime}(x) \\
-\left(\lambda^{2}+c \lambda\right) w(x)=0, \quad 0<x<1, \\
w(0)=w(1)=0 .
\end{gathered}
$$

Lemma 3. Let $\mathscr{A}$ be defined by (10); then $\operatorname{Re} \lambda<0$ for any $\lambda \in \sigma_{p}(\mathscr{A})$ and $\lambda \neq-b_{i}, i=1,2, \ldots, N$.

Proof. Firstly, we show that $\operatorname{Re} \lambda \leq 0$. When $\lambda \in \sigma_{p}(\mathscr{A}), \lambda \neq-$ $b_{i}, i=1,2, \ldots, N,(29)$ gives

$$
a^{2} w^{\prime \prime}(x)+\sum_{i=1}^{N} \frac{a_{i}}{\lambda+b_{i}} w^{\prime}(x)-\left(\lambda^{2}+c \lambda\right) w(x)=0 .
$$

Multiply by $\bar{w}$, the conjugate of $w$, and integrate over $[0,1]$ with respect to $x$ for the equation to give

$$
\begin{gathered}
a^{2} \int_{0}^{1} w^{\prime \prime}(x) \bar{w}(x) d x+\sum_{i=1}^{N} \frac{a_{i}}{\lambda+b_{i}} \int_{0}^{1} w^{\prime}(x) \bar{w}(x) d x \\
-\left(\lambda^{2}+c \lambda\right) \int_{0}^{1}|w(x)|^{2} d x=0 .
\end{gathered}
$$

That is,

$$
a^{2} \int_{0}^{1}\left|w^{\prime}(x)\right|^{2} d x+\left(\lambda^{2}+c \lambda\right) \int_{0}^{1}|w(x)|^{2} d x=0 .
$$

Replace $\lambda=\alpha+i \beta, \alpha, \beta \in \mathscr{R}$ in the above formula to get

$$
\begin{gathered}
a^{2} \int_{0}^{1}\left|w^{\prime}(x)\right|^{2} d x+\left(\alpha^{2}-\beta^{2}+c \alpha\right) \int_{0}^{1}|w(x)|^{2} d x \\
+i(2 \alpha \beta+c \beta) \int_{0}^{1}|w(x)|^{2} d x=0 .
\end{gathered}
$$

Let the real and imaginary parts of the equation equal zero to obtain

$$
\begin{gathered}
a^{2}\left\|w^{\prime}\right\|_{L^{2}}^{2}+\left(\alpha^{2}-\beta^{2}+c \alpha\right)\|w\|_{L^{2}}^{2}=0, \\
(2 \alpha \beta+c \beta)\|w\|_{L^{2}}^{2}=0 .
\end{gathered}
$$


If $\beta=0$, from the first equation of (34), then it has

$$
\alpha=-\frac{a^{2}\left\|w^{\prime}\right\|_{L^{2}}^{2}+\alpha^{2}\|w\|_{L^{2}}^{2}}{c\|w\|_{L^{2}}^{2}} \leq 0 .
$$

If $\beta \neq 0$, from the second equation of (34), then we have

$$
(2 \alpha+c)\|w\|_{L^{2}}^{2}=0
$$

so

$$
\alpha=-\frac{c}{2}<0
$$

Secondly, we prove that $\operatorname{Re} \lambda \neq 0$. We use contradiction argument. Suppose that $\operatorname{Re} \lambda=0, \lambda \in \sigma_{p}(\mathscr{A})$; letting $\lambda=$ $i \tau, \tau \in \mathscr{R}(\tau \neq 0)$, from (34), we have

$$
\begin{gathered}
a^{2}\left\|w^{\prime}\right\|_{L^{2}}^{2}-\tau^{2}\|w\|_{L^{2}}^{2}=0, \\
c \tau\|w\|_{L^{2}}^{2}=0 .
\end{gathered}
$$

From the second equation, we obtain $\tau=0$ or $\|w\|=0$. If $\|w\|=0$ holds, it has $w(x) \equiv 0$; then, from (28), we have $v(x)=\varphi_{i}(x)=0, i=1, \ldots, N$. Hence, it has only $\tau=0$. This is a contradiction. The proof is complete.

Now, we give the asymptotic fundamental solutions of (29) as $|\lambda| \rightarrow \infty$.

Lemma 4. Suppose that $0 \neq \lambda \in \sigma_{p}(\mathscr{A})$ and $\lambda \neq-b_{i}, i=$ $1,2, \ldots, N, x \in[0,1]$, where $\sigma_{p}(\mathscr{A})$ stands for the point spectrum set of $\mathscr{A}$. Then $e^{(\lambda / a) x}$ and $e^{-(\lambda / a) x}$ are linearly independent fundamental solutions of $w^{\prime \prime}(x)-\left(\lambda^{2} / a^{2}\right) w(x)=$ 0 , and

$$
w^{\prime \prime}(x)+\left(\frac{1}{a^{2}} \sum_{i=1}^{N} \frac{a_{i}}{\lambda+b_{i}}\right) w^{\prime}(x)-\frac{\lambda^{2}+c \lambda}{a^{2}} w(x)=0,
$$

as $|\lambda| \rightarrow \infty$ has the following asymptotic fundamental solutions:

$$
\begin{aligned}
& w_{1}(x)=e^{(\lambda / a) x}\left[w_{10}(x)+w_{11}(x) \lambda^{-1}\right]+\mathcal{O}\left(\lambda^{-2}\right), \\
& w_{2}(x)=e^{-(\lambda / a) x}\left[w_{20}(x)+w_{21}(x) \lambda^{-1}\right]+\mathcal{O}\left(\lambda^{-2}\right),
\end{aligned}
$$

where

$$
\begin{array}{r}
w_{10}(x)=e^{(c / 2 a) x}, \quad w_{11}(x)=-\left(\frac{c^{2}}{8 a}+\frac{k}{2 a^{2}}\right) x e^{(c / 2 a) x}, \\
w_{20}(x)=e^{-(c / 2 a) x}, \quad w_{21}(x)=\left(\frac{c^{2}}{8 a}-\frac{k}{2 a^{2}}\right) x e^{-(c / 2 a) x}, \\
k=\sum_{i=1}^{N} a_{i}>0 .
\end{array}
$$

Proof. The first claim is trivial. We only need to show that (40) is the asymptotic fundamental solution of (39). This can be done along the same way of Birkhoff [16] and Naimark [17]. Here we present briefly a simple calculation to (40). Let

$$
\begin{aligned}
& \widetilde{w_{1}}(x, \lambda):=e^{(\lambda / a) x}\left[w_{10}(x)+\frac{w_{11}(x)}{\lambda}\right], \\
& \widetilde{w_{2}}(x, \lambda):=e^{-(\lambda / a) x}\left[w_{20}(x)+\frac{w_{21}(x)}{\lambda}\right],
\end{aligned}
$$

where $w_{j 0}(x)$ and $w_{j 1}(x), j=1,2$, are some functions to be determined, and

$$
\mathscr{D}(w)=w^{\prime \prime}(x)+\frac{1}{a^{2}}\left(\sum_{i=1}^{N} \frac{a_{i}}{\lambda+b_{i}}\right) w^{\prime}(x)-\frac{\lambda^{2}+c \lambda}{a^{2}} w(x) .
$$

Substitute $\widetilde{w_{1}}(x, \lambda)$ and $\widetilde{w_{2}}(x, \lambda)$ into $\mathscr{D}(w)$, respectively, to yield

$$
\begin{aligned}
& e^{-(\lambda / a) x} \mathscr{D}\left(\widetilde{w_{1}}(x, \lambda)\right) \\
& =\frac{2 \lambda}{a}\left[w_{10}^{\prime}(x)+w_{11}^{\prime}(x) \lambda^{-1}\right]+\left[w_{10}^{\prime \prime}(x)+w_{11}^{\prime \prime}(x) \lambda^{-1}\right] \\
& -\frac{c \lambda}{a^{2}}\left[w_{10}(x)+w_{11}(x) \lambda^{-1}\right] \\
& +\frac{\lambda}{a^{3}}\left(\sum_{i=1}^{N} \frac{a_{i}}{\lambda+b_{i}}\right)\left[w_{10}(x)+w_{11}(x) \lambda^{-1}\right] \\
& +\frac{1}{a^{2}}\left(\sum_{i=1}^{N} \frac{a_{i}}{\lambda+b_{i}}\right)\left[w_{10}^{\prime}(x)+w_{11}^{\prime}(x) \lambda^{-1}\right] \\
& =\lambda\left[\frac{2}{a} w_{10}^{\prime}(x)-\frac{c}{a} w_{10}(x)\right] \\
& +\left[w_{10}^{\prime \prime}(x)+\frac{\sum_{i=1}^{N} a_{i}}{a^{3}} w_{10}(x)+\frac{2}{a} w_{11}^{\prime}(x)-\frac{c}{a^{2}} w_{11}(x)\right] \\
& +\lambda^{-1}\left[w_{11}^{\prime \prime}(x)-\frac{1}{a^{3}}\left(\sum_{i=1}^{N} \frac{a_{i} b_{i}}{1+\lambda^{-1} b_{i}}\right) w_{10}(x)\right. \\
& \left.+\frac{1}{a^{3}}\left(\sum_{i=1}^{N} \frac{a_{i}}{1+\lambda^{-1} b_{i}}\right) w_{11}(x)\right] \\
& +\lambda^{-1}\left[\frac{1}{a^{2}}\left(\sum_{i=1}^{N} \frac{a_{i}}{1+\lambda^{-1} b_{i}}\right) w_{10}^{\prime}(x)\right. \\
& \left.+\frac{1}{a^{2}}\left(\sum_{i=1}^{N} \frac{a_{i}}{\lambda+b_{i}}\right) w_{11}^{\prime}(x)\right] \\
& =\lambda\left[\frac{2}{a} w_{10}^{\prime}(x)-\frac{c}{a} w_{10}(x)\right] \\
& +\left[w_{10}^{\prime \prime}(x)+\frac{\sum_{i=1}^{N} a_{i}}{a^{3}} w_{10}(x)+\frac{2}{a} w_{11}^{\prime}(x)-\frac{c}{a^{2}} w_{11}(x)\right] \\
& +\lambda^{-1} G_{1}(x, \lambda)
\end{aligned}
$$




$$
\begin{aligned}
& e^{(\lambda / a) x} \mathscr{D}\left(\widetilde{w_{2}}(x, \lambda)\right) \\
& =-\frac{2 \lambda}{a}\left[w_{20}^{\prime}(x)+w_{21}^{\prime}(x) \lambda^{-1}\right] \\
& +\left[w_{20}^{\prime \prime}(x)+w_{21}^{\prime \prime}(x) \lambda^{-1}\right]-\frac{c \lambda}{a^{2}}\left[w_{20}(x)+w_{21}(x) \lambda^{-1}\right] \\
& -\frac{\lambda}{a^{3}}\left(\sum_{i=1}^{N} \frac{a_{i}}{\lambda+b_{i}}\right)\left[w_{20}(x)+w_{21}(x) \lambda^{-1}\right] \\
& +\frac{1}{a^{2}}\left(\sum_{i=1}^{N} \frac{a_{i}}{\lambda+b_{i}}\right)\left[w_{20}^{\prime}(x)+w_{21}^{\prime}(x) \lambda^{-1}\right] \\
& =\lambda\left[-\frac{2}{a} w_{20}^{\prime}(x)-\frac{c}{a^{2}} w_{20}(x)\right] \\
& +\left[w_{20}^{\prime \prime}(x)-\frac{1}{a^{3}}\left(\sum_{i=1}^{N} a_{i}\right) w_{20}(x)\right. \\
& \left.-\frac{2}{a} w_{21}^{\prime}(x)-\frac{c}{a^{2}} w_{21}(x)\right] \\
& +\lambda^{-1}\left[w_{21}^{\prime \prime}(x)+\frac{1}{a^{3}}\left(\sum_{i=1}^{N} \frac{a_{i} b_{i}}{1+\lambda^{-1} b_{i}}\right) w_{20}(x)\right. \\
& \left.-\frac{1}{a^{3}}\left(\sum_{i=1}^{N} \frac{a_{i}}{1+\lambda^{-1} b_{i}}\right) w_{21}(x)\right] \\
& +\lambda^{-1}\left[\frac{1}{a^{2}}\left(\sum_{i=1}^{N} \frac{a_{i}}{1+\lambda^{-1} b_{i}}\right) w_{20}^{\prime}(x)\right. \\
& \left.+\frac{1}{a^{2}}\left(\sum_{i=1}^{N} \frac{a_{i}}{\lambda+b_{i}}\right) w_{21}^{\prime}(x)\right] \\
& =\lambda\left[-\frac{2}{a} w_{20}^{\prime}(x)-\frac{c}{a^{2}} w_{20}(x)\right] \\
& +\left[w_{20}^{\prime \prime}(x)-\frac{1}{a^{3}}\left(\sum_{i=1}^{N} a_{i}\right) w_{20}(x)\right. \\
& \left.-\frac{2}{a} w_{21}^{\prime}(x)-\frac{c}{a^{2}} w_{21}(x)\right] \\
& +\lambda^{-1} G_{2}(x, \lambda),
\end{aligned}
$$

where

$$
\begin{aligned}
G_{j}(x, \lambda)= & (-1)^{j} \sum_{i=1}^{N} \frac{a_{i} b_{i}}{a^{3}\left(1+\lambda^{-1} b_{i}\right)} w_{j 0}(x) \\
& +(-1)^{j-1} \sum_{i=1}^{N} \frac{a_{i}}{a^{3}\left(1+\lambda^{-1} b_{i}\right)} w_{j 1}(x) \\
& +\sum_{i=1}^{N} \frac{a_{i}}{a^{2}\left(1+\lambda^{-1} b_{i}\right)} w_{j 0}^{\prime}(x) \\
& +\sum_{i=1}^{N} \frac{a_{i}}{a^{2}\left(\lambda+b_{i}\right)} w_{j 1}^{\prime}(x)+w_{j 1}^{\prime \prime}(x), \quad j=1,2 .
\end{aligned}
$$

Moreover, there exists some positive constant $\gamma$ such that

$$
\left|G_{j}(x, \lambda)\right| \leq \gamma, \quad \forall x \in[0,1] .
$$

Thus, letting the coefficients of $\lambda^{1}$ and $\lambda^{0}$ be zero gives

$$
\begin{gathered}
\frac{2}{a} w_{10}^{\prime}(x)-\frac{c}{a} w_{10}(x)=0, \quad \frac{2}{a} w_{20}^{\prime}(x)+\frac{c}{a^{2}} w_{20}(x)=0, \\
w_{10}^{\prime \prime}(x)+\frac{k}{a^{3}} w_{10}(x)+\frac{2}{a} w_{11}^{\prime}(x)-\frac{c}{a^{2}} w_{11}(x)=0, \\
w_{20}^{\prime \prime}(x)-\frac{k}{a^{3}} w_{20}(x)-\frac{2}{a} w_{21}^{\prime}(x)-\frac{c}{a^{2}} w_{21}(x)=0,
\end{gathered}
$$

where $k$ is given by (41).

Now, use the condition $w_{j 0}(0)=1, w_{j 1}(0)=0, j=1,2$, to get

$$
\begin{array}{ll}
w_{10}(x)=e^{(c / 2 a) x}, & w_{11}(x)=-\left(\frac{c^{2}}{8 a}+\frac{k}{2 a^{2}}\right) x e^{(c / 2 a) x}, \\
w_{20}(x)=e^{-(c / 2 a) x}, & w_{21}(x)=\left(\frac{c^{2}}{8 a}-\frac{k}{2 a^{2}}\right) x e^{-(c / 2 a) x} .
\end{array}
$$

These are the expressions of (41). When $|\lambda|$ is large enough, we can obtain the linearly independent asymptotic fundamental solutions of (39) given by (40) (see [16]):

$$
\begin{aligned}
& w_{1}(x)=e^{(\lambda / a) x}\left[w_{10}(x)+\frac{w_{11}(x)}{\lambda}\right]+\mathcal{O}\left(\lambda^{-2}\right), \\
& w_{2}(x)=e^{-(\lambda / a) x}\left[w_{20}(x)+\frac{w_{21}(x)}{\lambda}\right]+\mathcal{O}\left(\lambda^{-2}\right) .
\end{aligned}
$$

The proof is complete.

Assume that $\lambda \neq 0$ and $\lambda \neq-b_{i}, i=1,2, \ldots, N$, and let

$$
w(x)=\xi w_{1}(x)+\eta w_{2}(x),
$$

where $w_{j}(x), j=1,2$, are given by (40). Then substitute the above into the boundary conditions of (29) to obtain

$$
\Delta(\lambda)[\xi, \eta]^{\top}=0,
$$

where

$$
\Delta(\lambda)=\left(\begin{array}{cc}
1 & 1 \\
w_{1}(1) & w_{2}(1)
\end{array}\right) .
$$

Hence (29) admits nontrivial solution if and only if

$$
\operatorname{det}(\Delta(\lambda))=0,
$$

and the eigenvalues of (29) are the zeros of (53). Notice that

$$
\begin{aligned}
\operatorname{det}(\Delta(\lambda))= & w_{2}(1)-w_{1}(1) \\
= & e^{-\lambda / a}\left[w_{20}(1)+w_{21}(1) \lambda^{-1}\right] \\
& -e^{\lambda / a}\left[w_{10}(1)+w_{11}(1) \lambda^{-1}\right]+\mathcal{O}\left(\lambda^{-2}\right) \\
= & e^{-\lambda / a}\left[e^{-c / 2 a}+b e^{-c / 2 a} \lambda^{-1}\right] \\
& -e^{\lambda / a}\left[e^{c / 2 a}-d e^{c / 2 a} \lambda^{-1}\right]+\mathcal{O}\left(\lambda^{-2}\right),
\end{aligned}
$$


where

$$
b=\frac{c^{2}}{8 a}-\frac{k}{2 a^{2}}, \quad d=\frac{c^{2}}{8 a}+\frac{k}{2 a^{2}},
$$

and $k$ is given in Lemma 4 .

Therefore $\operatorname{det}(\Delta(\lambda))=0$ produces

$$
\begin{aligned}
e^{-\lambda / a} & {\left[e^{-c / 2 a}+b e^{-c / 2 a} \lambda^{-1}\right] } \\
& -e^{\lambda / a}\left[e^{c / 2 a}-d e^{c / 2 a} \lambda^{-1}\right]+\mathcal{O}\left(\lambda^{-2}\right)=0,
\end{aligned}
$$

which further leads to

$$
e^{-\lambda / a} e^{-c / 2 a}-e^{\lambda / a} e^{c / 2 a}+\mathcal{O}\left(\lambda^{-1}\right)=0 .
$$

At last, the solutions of $e^{(2 \lambda+c) / a}-1=0$ are

$$
\widetilde{\lambda_{n}}=-\frac{c}{2}+a n \pi i, \quad n \in \mathbb{Z} .
$$

Apply the Rouché theorem to (57) to give the solutions of (57) as follows:

$$
\lambda_{n}=\widetilde{\lambda_{n}}+\mathcal{O}\left(n^{-1}\right)=-\frac{c}{2}+a n \pi i+\mathcal{O}\left(\frac{1}{n}\right), \quad n \in \mathbb{Z} .
$$

We summarize the above analysis as the following Theorem.

Theorem 5. The eigenvalues of (29) have the following asymptotic expressions:

$$
\lambda_{n}=-\frac{c}{2}+a n \pi i+\mathcal{O}\left(n^{-1}\right), \quad n \in \mathbb{Z},
$$

especially,

$$
\operatorname{Re} \lambda_{n} \longrightarrow-\frac{c}{2}<0 \quad \text { as }|n| \longrightarrow \infty .
$$

That is, $\operatorname{Re} \lambda=-c / 2$ is the asymptote of the eigenvalues $\lambda_{n}$ given by (60). Furthermore, the corresponding eigenfunctions $w_{n}(x), n \in \mathbb{Z}$, have the asymptotic expressions

$$
\begin{gathered}
w_{n}(x)=\sin n \pi x+\mathcal{O}\left(n^{-1}\right), \\
\lambda_{n}^{-1} w_{n}(x)=-\frac{i}{a n \pi} \sin n \pi x+\mathcal{O}\left(n^{-1}\right) .
\end{gathered}
$$

Moreover, $\left\{w_{n}(x), n \in \mathbb{Z}\right\}$ and $\left\{\lambda_{n}^{-1} w_{n}(x), n \in \mathbb{Z}\right\}$ are approximately normalized in $L^{2}(0,1)$ in the sense that there exist positive constants $c_{1}$ and $c_{2}$ independent of $n$ such that, for $n \in \mathbb{Z}$,

$$
c_{1} \leq\left\|w_{n}\right\|_{L^{2}}, \quad\left\|\lambda_{n}^{-1} w_{n}\right\|_{L^{2}} \leq c_{2} .
$$

Proof. From above statement, (60) has been proved. We only need to show (62)-(63) to be valid. Since $\lambda \neq 0,-b_{i}, i=$ $1,2, \ldots, N$, in view of (52) and (60), Lemma 4 , and some facts in linear algebra, the eigenfunction $w$ corresponding to the eigenvalue $\lambda$ is given by

$$
\begin{aligned}
w(\lambda, x) & =\operatorname{det}\left(\begin{array}{cc}
1 & 1 \\
w_{1}(x) & w_{2}(x)
\end{array}\right)=w_{2}(x)-w_{1}(x) \\
& =e^{-(\lambda / a) x} e^{-(c / 2 a) x}-e^{(\lambda / a) x} e^{(c / 2 a) x}+\mathcal{O}\left(\lambda^{-1}\right) .
\end{aligned}
$$

Owing to the fact of (60) that

$$
\frac{\lambda_{n}}{a}+\frac{c}{2 a}=n \pi i+\mathcal{O}\left(n^{-1}\right), \quad n \in \mathbb{Z},
$$

(62) is hence proved by taking

$$
w_{n}(x)=\frac{1}{2} i w\left(\lambda_{n}, x\right) .
$$

Finally,

$$
\begin{aligned}
& \left\|w_{n}\right\|_{L^{2}}=\int_{0}^{1} \sin ^{2} n \pi x d x+\mathcal{O}\left(n^{-1}\right)=\frac{1}{2}+\mathcal{O}\left(n^{-1}\right), \\
& \left\|\lambda_{n}^{-1} w_{n}\right\|_{L^{2}} \\
& =\int_{0}^{1}\left(-\frac{i}{a n \pi} \sin n \pi x\right) \overline{\left(-\frac{i}{a n \pi} \sin n \pi x\right)} d x+\mathcal{O}\left(n^{-1}\right) \\
& =\frac{1}{2 a^{2} n^{2} \pi^{2}}+\mathcal{O}\left(n^{-1}\right) .
\end{aligned}
$$

These give (63). The proof is complete.

The following result is the consequence of Theorem 5 and Proposition 2.

Theorem 6. Let $\mathscr{A}$ be defined as in (10). Then

(i) $\mathscr{A}$ has the eigenvalues

$$
\left\{-b_{i}, i=1,2, \ldots, N\right\} \bigcup\left\{\lambda_{n}, \overline{\lambda_{n}}, n \in \mathcal{N}^{+}\right\},
$$

where $\lambda_{n 0}$ have the asymptotic expressions (60),

(ii) the eigenfunction corresponding to $-b_{i}$ is $e_{i+2}$ for any $i=1,2, \ldots, N$;

(iii) the eigenfunctions corresponding to $\lambda_{n}$ and $\overline{\lambda_{n}}$ are given by

$$
\begin{aligned}
W_{n 0}(x)= & \left(\frac{1}{n \pi} \sin n \pi x, i a \sin n \pi x, 0, \ldots, 0\right) \\
& +(0,1,1, \ldots, 1) \mathcal{O}\left(n^{-1}\right), \\
\overline{W_{n 0}}(x)= & \left(\frac{1}{n \pi} \sin n \pi x,-i a \sin n \pi x, 0, \ldots, 0\right) \\
& +(0,1,1, \ldots, 1) \mathcal{O}\left(n^{-1}\right),
\end{aligned}
$$

for $n \rightarrow \infty$, respectively, where $w_{n}(x)=$ $(1 / n \pi) \sin n \pi x, \lambda_{n} w_{n}(x)=i a \sin n \pi x+\mathcal{O}\left(n^{-1}\right)$ and $\varphi_{n}$ is given by

$$
\left(\varphi_{i}\right)_{n}(x)=\frac{a_{i}}{\lambda_{n}+b_{i}} w_{n}(x)=\mathcal{O}\left(n^{-1}\right) .
$$

Concerning about $\sigma(\mathscr{A})$, we have Theorem 7 .

Theorem 7. Let $\mathscr{A}$ be defined as in (10). Then $\sigma_{r}(\mathscr{A})$ and $\sigma_{c}(\mathscr{A})$ are empty sets, where $\sigma_{r}(\mathscr{A})$ and $\sigma_{c}(\mathscr{A})$ denote the set of residual and continuous spectrum of $\mathscr{A}$, respectively. 
Proof. We need only to prove that $\lambda \in \rho(\mathscr{A})$, when $\lambda \notin$ $\sigma_{p}(\mathscr{A})$. Letting $\lambda \notin \sigma_{p}(\mathscr{A})$, then $\lambda \neq-b_{i}, i=1,2, \ldots, N$. Solve $(\lambda I-\mathscr{A})\left(w, v, \varphi_{1}, \ldots, \varphi_{N}\right)=\left(\widetilde{w}, \widetilde{v}, \widetilde{\varphi_{1}}, \ldots, \widetilde{\varphi_{N}}\right)$ for $\widetilde{W}=$ $\left(\widetilde{w}, \widetilde{v}, \widetilde{\varphi_{1}}, \ldots, \widetilde{\varphi_{N}}\right) \in \mathscr{H}$ and $W=\left(w, v, \varphi_{1}, \ldots, \varphi_{N}\right) \in D(\mathscr{A})$; that is,

$$
\begin{gathered}
\lambda w(x)-v(x)=\widetilde{w}(x) \\
\lambda v(x)-\left[a^{2} w^{\prime \prime}(x)+\left(\sum_{i=1}^{N} \varphi_{i}(x)\right)^{\prime}-c v(x)\right]=\widetilde{v}(x) \\
\lambda \varphi_{i}(x)-\left(a_{i} w(x)-b_{i} \varphi_{i}(x)\right)=\widetilde{\varphi}_{i}(x) \\
w(0)=w(1)=0
\end{gathered}
$$

to get

$$
\begin{gathered}
v(x)=\lambda w(x)-\widetilde{w}(x), \\
\varphi_{i}(x)=\frac{1}{\lambda+b_{i}}\left(a_{i} w(x)+\widetilde{\varphi}_{i}(x)\right), \quad i=1, \ldots, N,
\end{gathered}
$$

and so

$$
\sum_{i=1}^{N} \varphi_{i}(x)=w(x) \sum_{i=1}^{N} \frac{a_{i}}{\lambda+b_{i}}+\sum_{i=1}^{N} \frac{\widetilde{\varphi_{i}}(x)}{\lambda+b_{i}} .
$$

Set

$$
\begin{aligned}
\theta(x) & =a^{2} w^{\prime}(x)+\sum_{i=1}^{N} \varphi_{i}(x) \\
& =a^{2} w^{\prime}(x)+w(x) \sum_{i=1}^{N} \frac{a_{i}}{\lambda+b_{i}}+\sum_{i=1}^{N} \frac{\widetilde{\varphi_{i}}(x)}{\lambda+b_{i}}
\end{aligned}
$$

Then we can write (71) as

$$
\begin{gathered}
\theta(x)=a^{2} w^{\prime}(x)+p_{1}(\lambda) w(x)+\widetilde{\varphi}(x), \\
\theta^{\prime}(x)=\left(\lambda^{2}+c \lambda\right) w(x)-\widetilde{v}(x)-(\lambda+c) \widetilde{w}(x), \\
w(0)=w(1)=0,
\end{gathered}
$$

where

$$
p(\lambda)=\sum_{i=1}^{N} \frac{a_{i}}{\lambda+b_{i}}, \quad \widetilde{\varphi}(x)=\sum_{i=1}^{N} \frac{\widetilde{\varphi_{i}}(x)}{\lambda+b_{i}} .
$$

Hence (75) can be rewritten as the following first-order system of differential equations:

$$
\begin{aligned}
& \frac{d}{d x}\left(\begin{array}{c}
w(x) \\
\theta(x)
\end{array}\right)=\left(\begin{array}{cc}
-\frac{p(\lambda)}{a^{2}} & \frac{1}{a^{2}} \\
\lambda^{2}+c \lambda & 0
\end{array}\right)\left(\begin{array}{c}
w(x) \\
\theta(x)
\end{array}\right) \\
&-\left(\begin{array}{c}
\frac{1}{a^{2}} \widetilde{\varphi}(x) \\
\widetilde{v}(x)+(\lambda+c) \widetilde{w}(x)
\end{array}\right), \\
& w(0)=w(1)=0 .
\end{aligned}
$$

Let

$$
A(\lambda)=\left(\begin{array}{cc}
-\frac{p(\lambda)}{a^{2}} & \frac{1}{a^{2}} \\
\lambda^{2}+c \lambda & 0
\end{array}\right)
$$

Then it has

$$
e^{A(\lambda) x}=\left(\begin{array}{ll}
a_{11}(\lambda, x) & a_{12}(\lambda, x) \\
a_{21}(\lambda, x) & a_{22}(\lambda, x)
\end{array}\right)
$$

where

$$
\begin{gathered}
a_{11}(\lambda, x)=-\frac{p(\lambda)}{2 \sqrt{q(\lambda)}} \Phi(\lambda, x)+\frac{1}{2} \Psi(\lambda, x), \\
a_{12}(\lambda, x)=\frac{1}{\sqrt{q(\lambda)}} \Phi(\lambda, x),
\end{gathered}
$$

$$
a_{21}(\lambda, x)=\frac{a^{2}\left(\lambda^{2}+c \lambda\right)}{\sqrt{q(\lambda)}} \Phi(\lambda, x)
$$

$$
a_{22}(\lambda, x)=\frac{p(\lambda)}{2 \sqrt{q(\lambda)}} \Phi(\lambda, x)+\frac{1}{2} \Psi(\lambda, x)
$$

$$
\begin{aligned}
& \Phi(\lambda, x)=\left(e^{\left((-p(\lambda)+\sqrt{q(\lambda)}) / 2 a^{2}\right) x}-e^{\left((-p(\lambda)-\sqrt{q(\lambda)}) / 2 a^{2}\right) x}\right), \\
& \Psi(\lambda, x)=\left(e^{\left((-p(\lambda)+\sqrt{q(\lambda)}) / 2 a^{2}\right) x}+e^{\left((-p(\lambda)-\sqrt{q(\lambda)}) / 2 a^{2}\right) x}\right),
\end{aligned}
$$

and $q(\lambda)$ is given by

$$
q(\lambda)=p^{2}(\lambda)+4 a^{2}\left(\lambda^{2}+c \lambda\right)
$$

The general solution of (77) is then found to be

$$
\begin{aligned}
\left(\begin{array}{c}
w(x) \\
\theta(x)
\end{array}\right)= & e^{A(\lambda) x}\left(\begin{array}{c}
w(0) \\
\theta(0)
\end{array}\right) \\
& -\int_{0}^{x} e^{A(\lambda)(x-\tau)}\left(\begin{array}{c}
\frac{1}{a^{2}} \widetilde{\varphi}(\tau) \\
\widetilde{v}(\tau)+(\lambda+c) \widetilde{w}(\tau)
\end{array}\right) d \tau .
\end{aligned}
$$


Since $w(0)=0$, it has

$$
\begin{aligned}
& w(x)=a_{12}(\lambda, x) \theta(0) \\
&-\int_{0}^{x}\left[\frac{\widetilde{\varphi}(\tau)}{a^{2}} a_{11}(\lambda, x-\tau)\right. \\
&\left.\quad+a_{12}(\lambda, x-\tau)(\widetilde{v}(\tau)+(\lambda+c) \widetilde{w}(\tau))\right] d \tau,
\end{aligned}
$$

$$
\begin{aligned}
\theta(x)=a_{22}(\lambda, x) \theta(0) \\
-\int_{0}^{x}\left[\frac{\tilde{\varphi}(\tau)}{a^{2}} a_{21}(\lambda, x-\tau)\right. \\
\left.\quad+a_{22}(\lambda, x-\tau)(\widetilde{v}(\tau)+(\lambda+c) \widetilde{w}(\tau))\right] d \tau .
\end{aligned}
$$

When $\widetilde{W}=\left(\widetilde{w}, \widetilde{v}, \widetilde{\varphi_{1}}, \ldots, \widetilde{\varphi_{N}}\right)=0,(83)$ and (84) reduce to the eigenvalue problem

$$
w(x)=a_{12}(\lambda, x) \theta(0), \quad \theta(x)=a_{22}(\lambda, x) \theta(0) .
$$

So, when $\lambda \in \sigma_{p}(\mathscr{A}), \lambda \neq-b_{i}, i=1,2, \ldots, N$, if and only if $a_{12}(\lambda, 1)=0$, that is,

$$
\frac{1}{\sqrt{q(\lambda)}}\left(e^{(-p(\lambda)+\sqrt{q(\lambda)}) / 2 a^{2}}-e^{(-p(\lambda)-\sqrt{q(\lambda)}) / 2 a^{2}}\right)=0
$$

which yields

$$
e^{(-p(\lambda)+\sqrt{q(\lambda)}) / 2 a^{2}}-e^{(-p(\lambda)-\sqrt{q(\lambda)}) / 2 a^{2}}=0
$$

This is the characteristic determinant of $\mathscr{A}$, which has the asymptotic form given by (60).

Now, since $\lambda \notin \sigma_{p}(\mathscr{A})$, it has

$$
\begin{aligned}
& a_{12}(\lambda, 1) \\
& =\frac{1}{\sqrt{q(\lambda)}}\left(e^{(-p(\lambda)+\sqrt{q(\lambda)}) / 2 a^{2}}-e^{(-p(\lambda)-\sqrt{q(\lambda)}) / 2 a^{2}}\right) \neq 0 .
\end{aligned}
$$

By the boundary condition $w(1)=0$, we have

$$
\begin{aligned}
\theta(0)= & \frac{1}{a_{12}(\lambda, 1)} \\
& \quad \times \int_{0}^{1}\left[\frac{\widetilde{\varphi}(\tau)}{a^{2}} a_{11}(\lambda, 1-\tau)\right. \\
& \left.\quad+a_{12}(\lambda, 1-\tau)(\widetilde{v}(\tau)+(\lambda+c) \widetilde{w}(\tau))\right] d \tau .
\end{aligned}
$$

Hence $w(x)$ is uniquely determined by (83) and $w^{\prime} \epsilon$ $L^{2}(0,1)$. Once $w$ is known, $v(x)$ and $\varphi_{i}(x)$ are also uniquely determined by (72). So $(\lambda I-\mathscr{A})^{-1}$ exists and is bounded. Therefore $\lambda \in \rho(\mathscr{A})$. The proof is complete.

\section{Spectrum-Determined Growth Condition and Exponential Stability}

Now we are in a position to consider the main result of this part, the so-called spectrum-determined growth condition for system (11), which is one of the most hard problems for infinite-dimensional systems. Our proof is based on the following characterization condition (see [18, Corollary 3.40, p.144]).

Lemma 8. Let $T(t)$ be a $C_{0}$-semigroup on a Hilbert space $H$ with its generator $A$. Let $\omega(A)$ be the growth bound of $T(t)$ and let

$$
s(A):=\sup \{\operatorname{Re} \lambda \mid \lambda \in \sigma(A)\}
$$

be the spectral bound of $A$. Then

$$
\begin{gathered}
\omega(A)=\inf \left\{\omega>s(A) \mid \sup _{\beta \in \mathscr{R}}\|R(\alpha+i \beta, A)\|\right. \\
\left.<M_{\alpha}<\infty, \forall \alpha \geq \omega\right\} .
\end{gathered}
$$

We also need Lemma 1.2 of [19].

Lemma 9. Let

$$
D(\lambda):=1+\sum_{i=1}^{n} P_{i}(\lambda) e^{\alpha_{i} \lambda}
$$

where $P_{i}(\lambda)$ are polynomials of $\lambda, \alpha_{i}$ are some complex numbers, and $n$ is a positive integer. Then, for all $\lambda$ outside those circles of radius $\varepsilon>0$ that centered at the zeros of $D(\cdot)$, one has

$$
|D(\lambda)| \geq C(\varepsilon)>0
$$

for some constant $C(\varepsilon)$ that depends only on $\varepsilon$.

Lemma 10. Let

$$
D_{0}(\lambda):=e^{\left((-p(\lambda)+\sqrt{q(\lambda)}) / 2 a^{2}\right) x}-e^{\left((-p(\lambda)-\sqrt{q(\lambda)}) / 2 a^{2}\right) x},
$$

where $p(\lambda)$ and $q(\lambda)$ are given by (76) and (81), respectively. Then, as indicated by (87), all eigenvalues $\lambda_{n}$ claimed by (60) are zeros of $D_{0}(\lambda)$. Moreover, for all $\lambda$ that are outside those circles centered at $\lambda_{n}$ with radius $\varepsilon>0$, one has

$$
\left|D_{0}(\lambda)\right| \geq e^{\operatorname{Re} \lambda / a} C_{0}(\varepsilon)>0,
$$

for some constant $C_{0}(\varepsilon)$ that depends only on $\varepsilon$.

Proof. By (57), it has

$$
\left|D_{0}(\lambda)\right|=-e^{\lambda / a} e^{c / 2 a}\left[1-e^{-2 \lambda / a} e^{-c / a}+\mathcal{O}\left(\lambda^{-1}\right)\right] .
$$


Then the desired result follows from Lemma 9. The proof is complete.

Theorem 11. Let $\mathscr{A}$ be defined by (10). Then the spectrumdetermined growth condition holds true for $e^{\mathscr{A} t}: s(\mathscr{A})=\omega(\mathscr{A})$.

Proof. By Lemma 8, the proof will be accomplished if we can prove that, for any $\lambda \neq 0$ and $\lambda=\alpha+i \beta \in \mathscr{C}$ with $\alpha \geq \omega>$ $s(\mathscr{A})$ and $\beta \in \mathscr{R}$, there exists a constant $M_{\alpha}$ such that

$$
\sup _{\beta \in \mathscr{R}}\|R(\alpha+i \beta, \mathscr{A})\| \leq M_{\alpha}<\infty .
$$

Let $\lambda=\alpha+i \beta \in \mathscr{C}$ with $\alpha \geq \omega>s(\mathscr{A})$ and $\beta \in \mathscr{R}$. For any $\widetilde{Y}=\left(\widetilde{w}, \widetilde{v}, \widetilde{\varphi_{1}}, \ldots, \widetilde{\varphi_{N}}\right) \in \mathscr{H}_{1}$, from the proof of Theorem 7 , $Y=R(\lambda, \mathscr{A}) \widetilde{Y}=\left(w, v, \varphi_{1}, \ldots, \varphi_{N}\right) \in D(\mathscr{A})$ satisfies

$$
\begin{aligned}
& w(x)=a_{12}(\lambda, x) \theta(0) \\
& -\int_{0}^{x}\left[\frac{\widetilde{\varphi}(\tau)}{a^{2}} a_{11}(\lambda, x-\tau)\right. \\
& \left.+a_{12}(\lambda, x-\tau)(\widetilde{v}(\tau)+(\lambda+c) \widetilde{w}(\tau))\right] d \tau \\
& v(x)=\lambda w(x)-\widetilde{w}(x), \quad \widetilde{\varphi}(x)=\sum_{i=1}^{N} \frac{\widetilde{\varphi}_{i}(x)}{\lambda+b_{i}}, \\
& \varphi_{i}(x)=\frac{a_{i}}{\lambda+b_{i}} w(x)+\frac{1}{\lambda+b_{i}} \widetilde{\varphi}_{i}(x), \quad i=1,2, \ldots, N, \\
& \theta(0)=\frac{1}{a_{12}(\lambda, 1)} \\
& \times \int_{0}^{1}\left[\frac{\widetilde{\varphi}(\tau)}{a^{2}} a_{11}(\lambda, 1-\tau)\right. \\
& \left.+a_{12}(\lambda, 1-\tau)(\widetilde{v}(\tau)+(\lambda+c) \widetilde{w}(\tau))\right] d \tau,
\end{aligned}
$$

where $a_{1 k}(\lambda, x), k=1,2$, are given by $(80)$.

Firstly, it is easy to see that there is a positive constant $M_{1 \alpha}$ such that

$$
\left|\frac{p(\lambda)}{\sqrt{q(\lambda)}}\right| \leq M_{1 \alpha}<\infty .
$$

Secondly, for simplicity, let $d_{1}(\lambda)=\operatorname{Re}(-p(\lambda)+\sqrt{q(\lambda)})$ and $d_{2}(\lambda)=\operatorname{Im}(-p(\lambda)+\sqrt{q(\lambda)})$, since

$$
\begin{aligned}
& \int_{0}^{1} e^{ \pm\left((-p(\lambda)+\sqrt{q(\lambda)}) / 2 a^{2}\right) x} e^{ \pm\left((-p(\lambda)+\sqrt{q(\lambda)}) / 2 a^{2}\right)} x d x \\
& =\int_{0}^{1} e^{ \pm\left(1 / 2 a^{2}\right)[-p(\lambda)+\sqrt{q(\lambda)}+\overline{(-p(\lambda)+\sqrt{q(\lambda)})}] x} d x \\
& = \pm \frac{a^{2}}{d_{1}(\lambda)}\left(e^{ \pm d_{1}(\lambda) / a^{2}}-1\right) \text {, }
\end{aligned}
$$

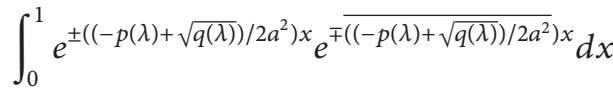

$$
\begin{aligned}
& =\int_{0}^{1} e^{ \pm\left(1 / 2 a^{2}\right)[-p(\lambda)+\sqrt{q(\lambda)}-\overline{(-p(\lambda)+\sqrt{q(\lambda)})}] x} d x \\
& = \begin{cases}1, & \beta=0, \\
\pm \frac{a^{2}}{i d_{2}(\lambda)}\left(e^{ \pm i d_{2}(\lambda) / a^{2}}-1\right), & \beta \neq 0,\end{cases} \\
& \left( \pm \frac{-p(\lambda)+\sqrt{q(\lambda)}}{2 a^{2}}\right)\left( \pm \frac{\overline{-p(\lambda)+\sqrt{q(\lambda)}}}{2 a^{2}}\right) \\
& \times \int_{0}^{1} e^{ \pm\left((-p(\lambda)+\sqrt{q(\lambda)}) / 2 a^{2}\right) x} e^{ \pm \overline{\left((-p(\lambda)+\sqrt{q(\lambda)}) / 2 a^{2}\right)} x} d x \\
& = \pm \frac{a^{2}\left(d_{1}^{2}(\lambda)+d_{2}^{2}(\lambda)\right)}{d_{1}(\lambda)}\left(e^{ \pm d_{1}(\lambda) / a^{2}}-1\right), \\
& \left( \pm \frac{-p(\lambda)+\sqrt{q(\lambda)}}{2 a^{2}}\right)\left(\mp \frac{\overline{-p(\lambda)+\sqrt{q(\lambda)}}}{2 a^{2}}\right) \\
& \times \int_{0}^{1} e^{ \pm\left((-p(\lambda)+\sqrt{q(\lambda)}) / 2 a^{2}\right) x} e^{\mp\left(\left((-p(\lambda)+\sqrt{q(\lambda)}) / 2 a^{2}\right)\right.} x d x \\
& = \begin{cases}-\left(d_{1}^{2}(\lambda)+d_{2}^{2}(\lambda)\right), & \beta=0, \\
\pm \frac{a^{2}\left(d_{1}^{2}(\lambda)+d_{2}^{2}(\lambda)\right)}{i d_{2}(\lambda)}\left(e^{ \pm i d_{2}(\lambda) / a^{2}}-1\right), & \beta \neq 0 .\end{cases}
\end{aligned}
$$

So combing (99), there is a positive constant $M_{2 \alpha}$ such that

$$
\begin{array}{r}
\sup _{\beta \in \mathscr{R}}\left\|a_{j k}(\lambda, \cdot)\right\|_{L^{2}} \leq M_{2 \alpha}<\infty, \\
\sup _{\beta \in \mathscr{R}}\left\|a_{j k}^{\prime}(\lambda, \cdot)\right\|_{L^{2}} \leq M_{3 \alpha}<\infty, \\
1 \leq j, k \leq 2 .
\end{array}
$$

Thirdly, by Proposition 2 and Theorem 7, it has

$$
s(\mathscr{A})=\sup \{\operatorname{Re} \lambda \mid \lambda \in \sigma(\mathscr{A})\}=\sup \left\{\operatorname{Re} \lambda \mid \lambda \in \sigma_{p}(\mathscr{A})\right\} .
$$

Define

$$
\varepsilon_{\alpha}=\inf _{\lambda_{n} \in \sigma_{p}(\mathscr{A}), \beta \in \mathscr{R}}\left|\lambda_{n}-\alpha-i \beta\right|
$$


By Lemma 10, there exists a positive constant $C_{0}\left(\varepsilon_{\alpha}\right)$ depending only on $\alpha$ such that

$$
\begin{aligned}
\left|D_{0}(\lambda)\right| & =e^{\left((-p(\lambda)+\sqrt{q(\lambda)}) / 2 a^{2}\right) x}-e^{\left((-p(\lambda)-\sqrt{q(\lambda)}) / 2 a^{2}\right) x} \\
& \geq e^{\alpha / a} C_{0}\left(\varepsilon_{\alpha}\right)>0 .
\end{aligned}
$$

Hence, by (86), there exists a positive constant $M_{4 \alpha}$ depending only on $\alpha$ such that

$$
\left|\frac{1}{a_{12}(\lambda, 1)}\right| \leq M_{4 \alpha}<\infty .
$$

So, by the Cauchy-Schwartz inequality, it follows from (101) and the last equation of (98) that there is a positive constant $M_{5 \alpha}$ such that

$$
\sup _{\beta \in \mathscr{R}}|\theta(0)| \leq M_{5 \alpha}\|\tilde{Y}\|_{\mathscr{H}}<\infty
$$

Finally, from (98), there is a positive constant $M_{\alpha}$ such that

$$
\begin{aligned}
& \sup _{\beta \in \mathscr{R}}\|Y\|_{\mathscr{H}} \\
& =\sup _{\beta \in \mathscr{R}}\left\{a^{2}\left\|w^{\prime}\right\|_{L^{2}}+\|v\|_{L^{2}}+\sum_{i=1}^{N}\left(\left\|\varphi_{i}^{\prime}\right\|_{L^{2}}+\left\|\varphi_{i}\right\|_{L^{2}}\right)\right\} \\
& \leq M_{\alpha}\|\tilde{Y}\|_{\mathscr{H}}<\infty .
\end{aligned}
$$

This gives (97). The proof is complete.

The following result gives a strongly exponential stability for the system (1).

Theorem 12. $e^{\mathscr{A} t}$ is exponentially stable; that is, there exist constants $M>1, \omega>0$ such that

$$
\left\|e^{\mathscr{A} t}\right\| \leq M e^{-\omega t}
$$

or, equivalently,

$$
\begin{aligned}
E(t)=\frac{1}{2} \int_{0}^{1}\left[a^{2}\left|w_{x}(x, t)\right|^{2}+\left|w_{t}(x, t)\right|^{2}\right. & \\
& \left.+\sum_{i=1}^{N}\left(\left|\varphi_{i}^{\prime}(x, t)\right|^{2}+\left|\varphi_{i}(x, t)\right|^{2}\right)\right] d x \\
\leq & M e^{-\omega t} E(0)=\frac{M}{2} e^{-\omega t} \int_{0}^{1}\left(\left|w_{0}(x)\right|^{2}+\left|w_{1}(x)\right|^{2}\right) d x
\end{aligned}
$$

Proof. By the spectrum-determined growth condition claimed by Theorem 11, the asymptote for eigenvalues of (61) and (109), $e^{\mathscr{A} t}$, is exponentially stable if and only if

$$
\operatorname{Re} \lambda<0, \quad \forall \lambda \in \sigma(\mathscr{A}) .
$$

From Proposition 2, Lemma 3, and Theorem 7, for arbitrary, $\lambda \in \sigma(\mathscr{A}), \operatorname{Re} \lambda<0$. The proof is complete.

\section{Conflict of Interests}

The author declares that there is no conflict of interests regarding the publication of this paper.

\section{References}

[1] M. Fabrizio and B. Lazzari, "On the existence and the asymptotic stability of solutions for linearly viscoelastic solids," Archive for Rational Mechanics and Analysis, vol. 116, no. 2, pp. 139-152, 1991.

[2] K. S. Liu and Z. Y. Liu, "Exponential decay of energy of the Euler-Bernoulli beam with locally distributed Kelvin-Voigt damping," SIAM Journal on Control and Optimization, vol. 36, no. 3, pp. 1086-1098, 1998.

[3] K. S. Liu and Z. Y. Liu, "Exponential decay of energy of vibrating strings with local viscoelasticity," Zeitschrift für Angewandte Mathematik und Physik, vol. 53, no. 2, pp. 265-280, 2002.

[4] J. E. Muñoz Rivera and D. Andrade, "Exponential decay of nonlinear wave equation with a viscoelastic boundary condition," Mathematical Methods in the Applied Sciences, vol. 23, no. 1, pp. 41-61, 2000.

[5] B. Z. Guo, J. M. Wang, and G. D. Zhang, "Spectral analysis of a wave equation with Kelvin-Voigt damping," Zeitschrift für Angewandte Mathematik und Mechanik, vol. 90, no. 4, pp. 323$342,2010$.

[6] B.-Z. Guo and G. D. Zhang, "On spectrum and Riesz basis property for one-dimensional wave equation with Boltzmann damping," ESAIM: Control, Optimisation and Calculus of Variations, vol. 18, no. 3, pp. 889-913, 2012.

[7] J. M. Wang and B. Z. Guo, "On dynamic behavior of a hyperbolic system derived from a thermoelastic equation with memory type," Journal of the Franklin Institute, vol. 344, no. 2, pp. 7596, 2007.

[8] J. M. Wang, B. Z. Guo, and M. Y. Fu, "Dynamic behavior of a heat equation with memory," Mathematical Methods in the Applied Sciences, vol. 32, no. 10, pp. 1287-1310, 2009.

[9] S. P. Chen, K. S. Liu, and Z. Y. Liu, "Spectrum and stability for elastic systems with global or local Kelvin-Voigt damping," SIAM Journal on Applied Mathematics, vol. 59, no. 2, pp. 651668, 1999.

[10] L. Pandolfi, "Riesz systems and controllability of heat equations with memory," Integral Equations and Operator Theory, vol. 64, no. 3, pp. 429-453, 2009.

[11] V. Pivovarchik and C. Tretter, "Asymptotic separation of the spectrum of quadratic operator pencils associated with damped dynamical systems," Asymptotic Analysis, vol. 36, no. 1, pp. 1-12, 2003.

[12] C. Tretter, "A linearization for a class of $\lambda$-nonlinear boundary eigenvalue problems," Journal of Mathematical Analysis and Applications, vol. 247, no. 2, pp. 331-355, 2000.

[13] H. L. Zhao, K. S. Liu, and C. G. Zhang, "Stability for the Timoshenko beam system with local Kelvin-Voigt damping," Acta Mathematica Sinica (English Series), vol. 21, no. 3, pp. 655666, 2005.

[14] E. Zuazua, "Exponential decay for the semilinear wave equation with locally distributed damping," Communications in Partial Differential Equations, vol. 15, no. 2, pp. 205-235, 1990.

[15] A. Pazy, Semigroups of Linear Operators and Applications to Partial Differential Equations, vol. 44 of Applied Mathematical Sciences, Springer, Berlin, Germany, 1983. 
[16] G. D. Birkhoff, "On the asymptotic character of the solutions of certain linear differential equations containing a parameter," Transactions of the American Mathematical Society, vol. 9, no. 2, pp. 219-231, 1908.

[17] M. A. Naimark, Linear Differential Operators, vol. 1, Ungar, New York, NY, USA, 1967.

[18] Z. H. Luo, B. Z. Guo, and O. Morgul, Stability and Stabilization of Infinite Dimensional Systems with Applications, Communications and Control Engineering Series, Springer, London, UK, 1999.

[19] A. A. Shkalikov, "Boundary problems for ordinary differential equations with parameter in the boundary conditions," Journal of Soviet Mathematics, vol. 33, no. 6, pp. 1311-1342, 1986. 


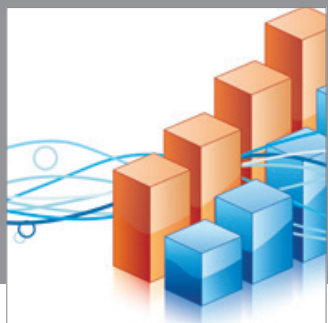

Advances in

Operations Research

mansans

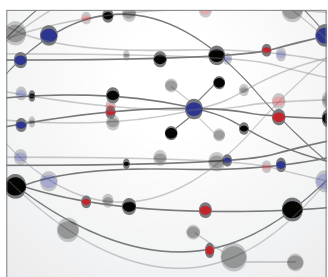

The Scientific World Journal
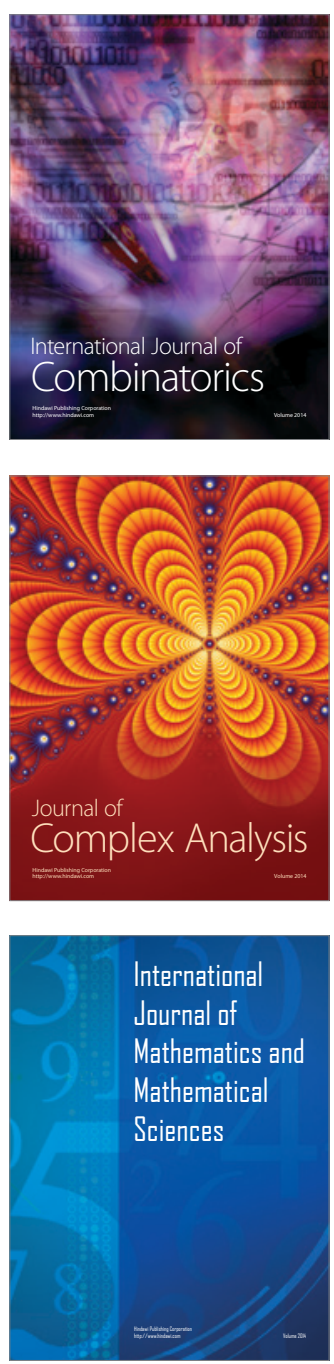
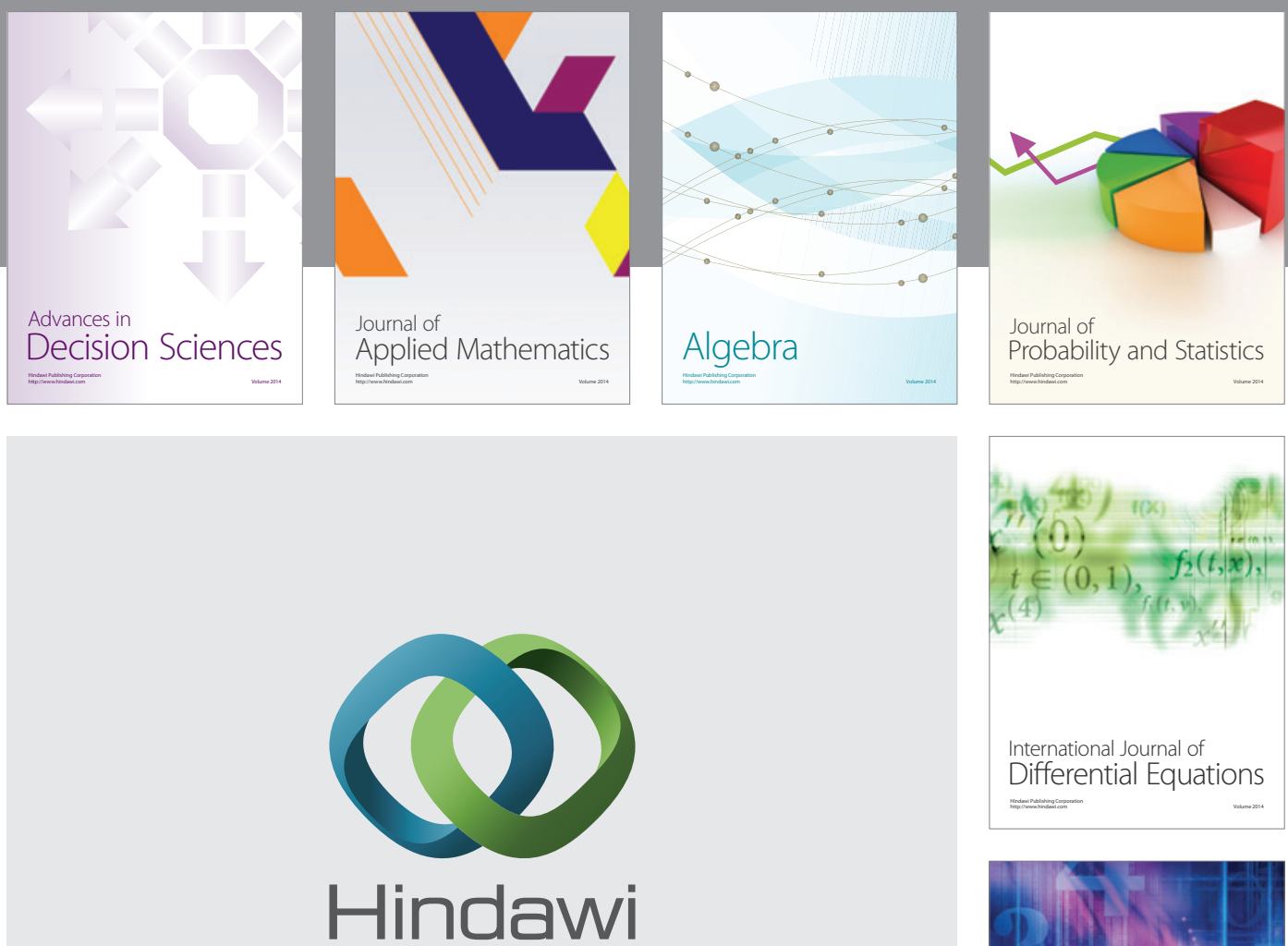

Submit your manuscripts at http://www.hindawi.com
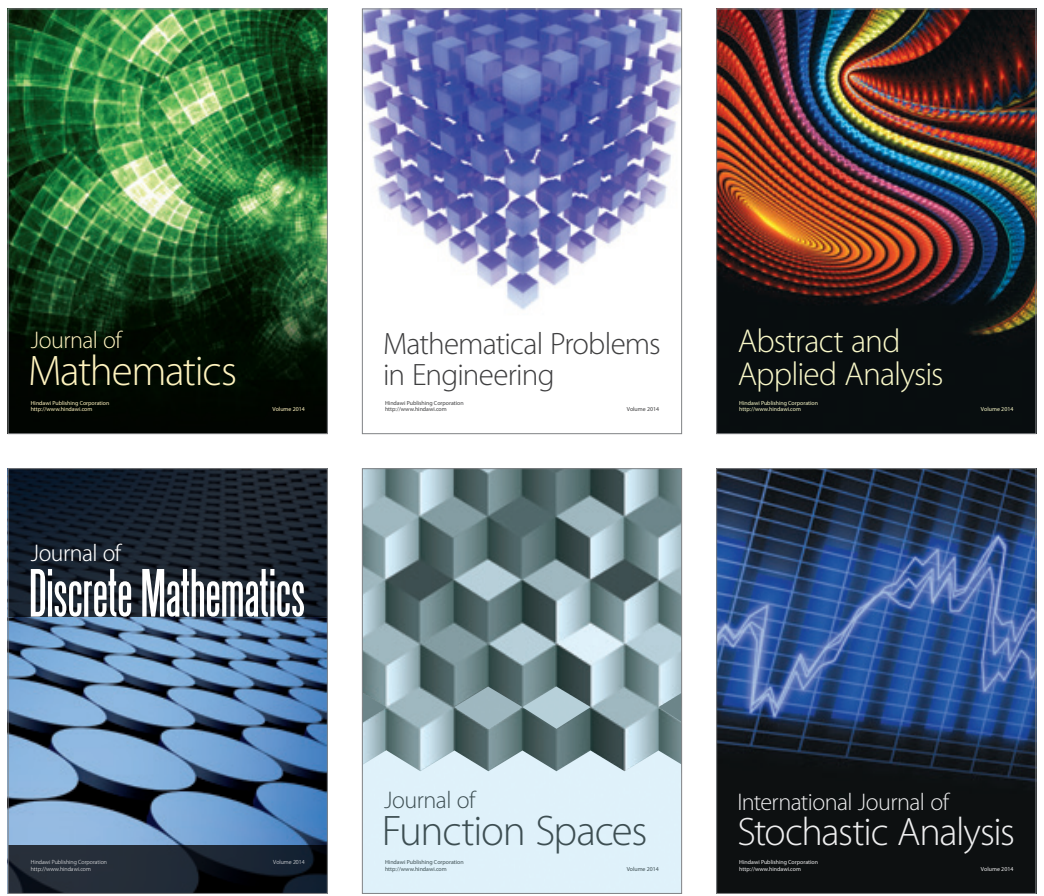

Journal of

Function Spaces

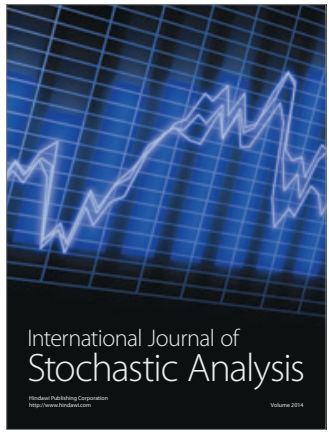

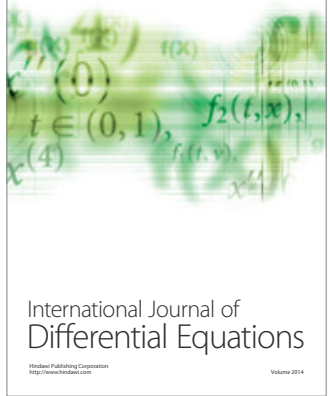
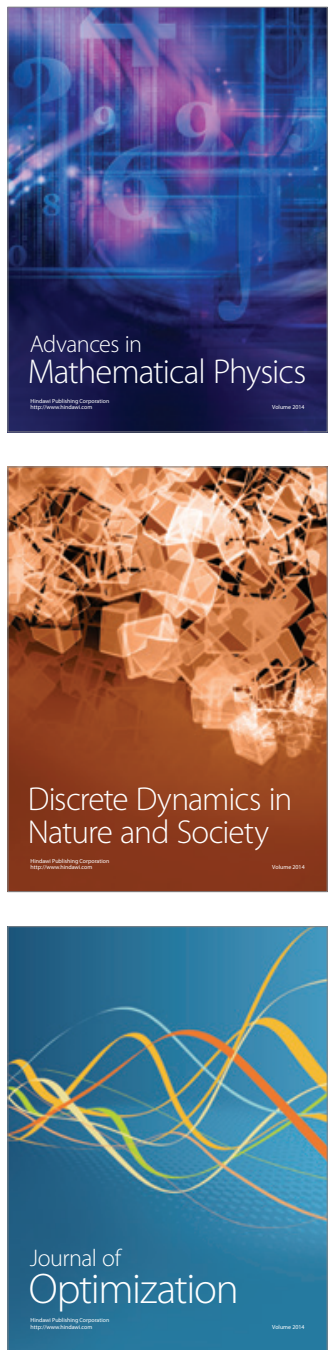